\title{
Peizoresistive Mems Cantilever based Co2 Gas Sensor
}

\author{
S. Subhashini \\ Research Scholar \\ Sathyabam University, Chennai
}

\author{
A. Vimala Juliet \\ HOD-EIE \\ SRM University, Chennai
}

\begin{abstract}
A study about the piezoresistive Micro-Electro-Mechanical Systems (MEMS) cantilever for a chemical sensitive - mass based sensor has been carried out to enhance sensor sensitivity. The sensitive region attracts the $\mathrm{CO} 2$ molecules there by introducing the stress concentration region (SCR). Three types of SCR geometry designs were first analysed using Intellisuite software to study the effect of stress and its distribution when varying mass is applied at the SCR. The results showed that the rectangular SCR design has the highest stress and showed a linear relation too when compared to the other types. Then, the length of rectangular SCR is varied to study the stress distribution along the cantilever. The piezoresistive element was then placed at various positions to obtain the highest stress resulting due to the deflection of the cantilever. The impact due the SCR was also analysed. The testing results of this piezoresistive MEMS cantilever with rectangular SCR had successfully enhanced sensitivity compared to the piezoresistive MEMS cantilever without SCR when varying mass is applied. Therefore this SCR approach appears to be suitable for enhancing the sensitivity of a massbased piezoresistive MEMS cantilever sensor.
\end{abstract}

\section{General Terms}

$\mathrm{CO} 2$ Gas Sensor using MEMS

\section{Keywords}

MEMS, chemical cantilever, peizoresistivity, stress, deflection, sensitivity

\section{INTRODUCTION}

The most prominent greenhouse gases in the Earth's atmosphere are water vapor, carbon dioxide, methane, nitrous oxide, ozone and CFCs. The alarming increase among these gases is that of $\mathrm{CO} 2$ and is a silent killer. Hence a sensor to monitor the quantity of $\mathrm{CO} 2$ molecules is necessary leading to this paper.

\footnotetext{
Miniaturization has gained enough significance as specified by Moores Law. The recent Micro-Electro-Mechanical Systems (MEMS) research efforts focus on several converging areas of science and technology such as biotechnology and health sciences. Brugger et al. have pointed out that the cantilever sensor is the simplest device among MEMS devices that offers a very promising future for the development of novel physical, chemical and biological sensors. The small size of MEMS allows for the construction of arrays of hundreds of sensor devices on a single substrate. By being capable of producing thousands of devices on each individual silicon wafer, the cost per unit can be driven down to affordable prices.
}

The bending of the cantilever is related to the applied mass or molecular substance that binds on the surface. The bending produces two effects 1) It changes the orientation of the cantilever surface with respect to the horizontal surface 2) It produces surface stress and hence strains. This stress could be converted to resistance and then converted to an equivalent voltage using a bridge circuit.

Several available detection methods have been used in the literature such as capacitance, optical and piezoresistive methods. The complicated capacitive system setup and laser heating effect of the optical laser has led to the selection and use of the piezoresistive method for the current study. Highly sensitive sensor devices are desired in many applications to increase the performance of detection. A sensor's sensitivity indicates how much the sensor's output changes when the quantity measured changes. This is owing to the small detection of biological mass to allow for readable results to be amplified. Instead of scaling down the device geometry, some studies have published many other approaches aimed at increasing the sensitivity of the MEMS cantilever. These approaches include changing the material used for cantilever structure that has a low Young's modulus by using SU-8 polymer and amplifying the readout systems. Among the increasing sensitivity approaches, introducing stress concentration region (SCR) seems to be the most suitable approach in increasing the sensitivity of MEMS cantilever since the piezoresistive detection is highly dependent on stress occurred at the cantilever. $\mathrm{Yu}$ et al. introduced holes to the beam in their simulation analysis to study the effect of surface stress on the sensitivity of MEMS cantilever. $\mathrm{He}$ and $\mathrm{Li}$ studied surface stress effect on various types of SCR holes and a number of holes using ANSYSw. They found that adding more holes to MEMS cantilevers did not help to enhance the surface stress. Bhatti et al. also completed similar studies and confirmed the findings that only one hole is sufficient to optimise the surface stress.

In this Paper, the SCR approach is taken to enhance the selectivity and sensitivity of the MEMS cantilever, and two types of piezoresistive MEMS cantilevers were designed and fabricated: the first is a piezoresistive MEMS cantilever without SCR and the second is a piezoresistive MEMS cantilever with rectangular SCR, since they can easily compensate for the environmental changes, in a wheatstone bridge. The proposed SCR designs are analysed using Intellisuite software to predict the design parametric study.

\section{STRUCTURE OF THE PROPOSED SYSTEM}

The main objective is to selectively choose the $\mathrm{CO} 2$ molecules from the mixture of gas molecules present in the atmosphere. This selection is done by using a polymer coating 
on top of the cantilever. The sensor designed for achieving this is as shown in Fig. 1. In Fig. 1(a) the sensing cantilever structure is shown and here we can see that a polymer coating is at the end to create the resistive changes based on the quantity of $\mathrm{CO} 2$. The gaseous inlet from the atmosphere is given to the entire setup and this exposure lets the $\mathrm{CO} 2$ molecules to be selectively adsorbed and thereby increasing the mass of the cantilever. This increase in mass results in a stress near the fixed end and could be sensed using a peizoresistive element placed there and which will form an arm of the wheatstone bridge as shown in Fig. 2. This change in mass of the cantilever due to the $\mathrm{CO} 2$ molecules adsorption results in a change in the resistance thereby resulting in the bridge imbalance giving a voltage output. This change in voltage is noted and can be interpreted with the quantity of $\mathrm{CO} 2$ molecules adsorbed and hence the quantity of $\mathrm{CO} 2$ present in the atmosphere.

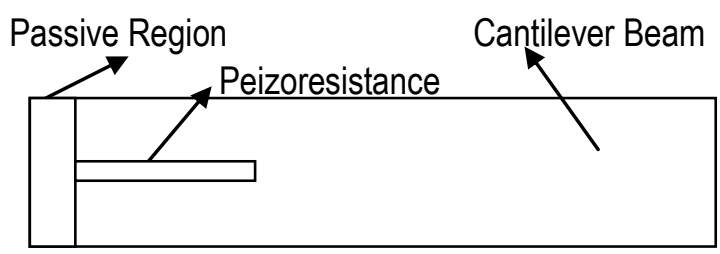

Fig 1(a): The basic sensing cantilever structure

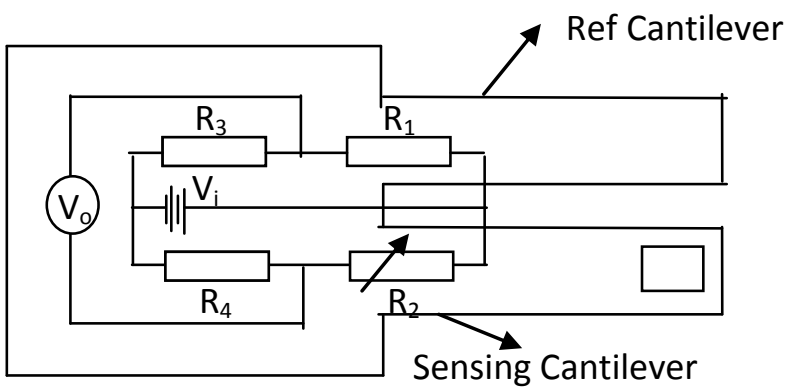

Fig 1(b): The basic reference cantilever structure

The wheatstone bridge setup is a well known one to find the resistance change. The change in piezoresistor is linearly related to the applied stress and the strain developed $(9,11,12)$. The resistance change is found using the bridge circuit made up of a combination of four peizoresistors as shown in Fig.2. Initially when no $\mathrm{CO} 2$ molecules are adsorbed the bridge is balanced giving out zero output voltage. This implies that the reference cantilever's peizoresistor equals that of the sensing cantilever's peizoresistor, this is advantageous as the environmental effects can be neutralized. Later as the Co2 molecules get adsorbed on the surface the mass of the cantilever increases and thereby deflects from its initial position resulting in a strain. The molecular weight of $\mathrm{CO} 2$ is $44.01 \mathrm{~g} / \mathrm{mol}$ and this creates a force acting on the cantilever. The peizoresistor of the sensing cantilever thereby changes resulting in a imbalance of the bridge and hence produces a voltage.
Fig 2: The wheatstone bridge circuit with the reference and sensing cantilevers

\section{FABRICATION STEPS FOR THE PEIZORESISTIVE GAS SENSOR}

The simulation study was conducted for the peizoresistive sensor using the Intellisuite software. The fabrication of the sensor was done effectively using step by step process of deposition and patterning using proper mask layers and definitions and etching process. It is at this stage where the size of the cantilever, the sensing area and the peizoresistor are defined. Surface micromachining and bulk micromachining methods are employed in patterning. The stepwise process followed is given below.

1. Define $\mathrm{Si}<100>$

2. Deposition of $\mathrm{SiO} 2$

3. Deposition of Photoresist (PR-S1800)

4. Definition of UV

5. Partially Etch Photoresist

6. Partially etch $\mathrm{SiO} 2$

7. Partially etch the photoresist

8. Deposition of Poly silicon

9. Deposition of photoresist

10. Definition of UV

11. Etch through photoresist

12. Etch through Poly silicon

13. Partially etch photoresist

14. Deposition of silicon

15. Deposit $\mathrm{ZnO}$

16. Deposit photoresist

17. Definition UV

18. Etch through photoresist

19. Etch through $\mathrm{ZnO}$

20. Partially etch photoresist

21. Etch polysilicon

The sensor thus efficiently fabricated is exported to the thermo electro mechanical analyser where the various analysis are done to study the effective performance. The final product will resemble fig. 2 .

\section{SIMULATION RESULTS}

The fabricated sensor after being transferred to the Thermo Electro mechanical Analyser is now fit to take up the boundary conditions and the inputs. Proper meshing is done to save time and in the meantime not to miss out the values. Hence the optimisations are done to have a better selectivity and sensitivity. 


\subsection{Mesh sizing:}

Mesh size determines the analysis segment. This implies as the mesh size decreases the execution time will be more and vice versa. In the mean time inorder to reduce the running time if we go in for increased mesh size sometimes depending on the process we might loose some minute important datas, this can not be tolerated. Hence we will have to obtain an optimum mesh size to efficiently run the process. Few theoretical calculations were made and compared with the simulated response to get the optimum settings. Fig.3 illustrates the relation between the mesh size and the displacement for the same sized cantilever, boundary conditions and input. It was observed that when the mesh size was 80 the theoretical results matched with the simulation results and it was also observed that the linearity criteria was fulfilled about that region. Hence 80 was choosen as the mesh size.

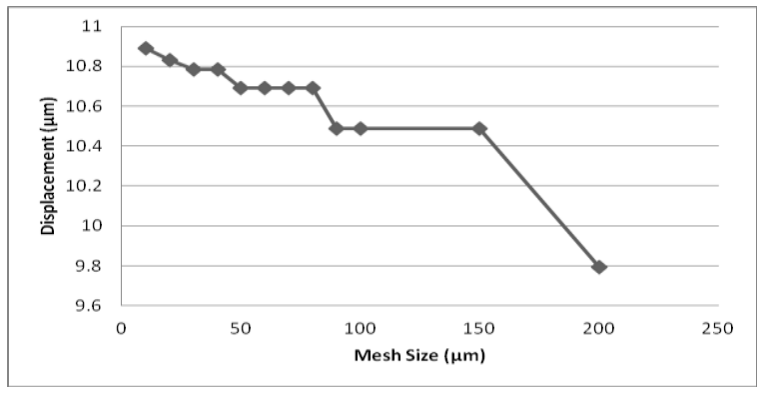

Fig 3: Characteristic curve to determine the mesh size

\subsection{SCR Design}

The sensitive area plays an very important role in the determination of the $\mathrm{CO} 2$ quantity. Hence few shapes were considered and the best option is considered further as an ideal shape. Fig. 4 shows the placement of the sensitive layer.

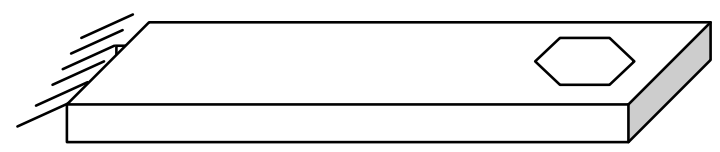

Fig 4(a): Cantilever with hexagonal SCR Design

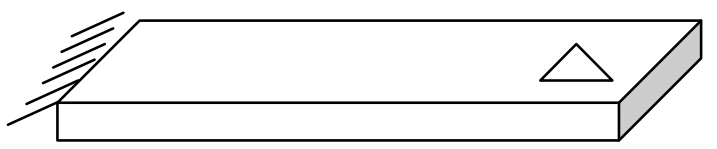

Fig 4(b): Cantilever with triangular SCR Design

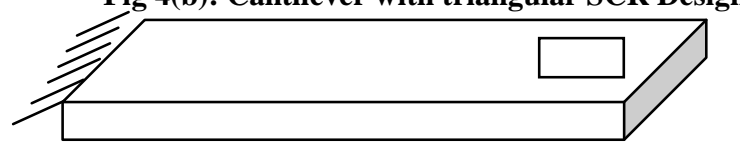

Fig 4(c): Cantilever with rectangular SCR Design
Having designed the shapes the input pressure is applied and the maximum displacement produced by the cantilever is measured and plotted. From the graph it is seen that the rectangular shaped SCR produced more linear effect and hence we shall stick on with rectangular SCR. This is visualized in Fig. 5.

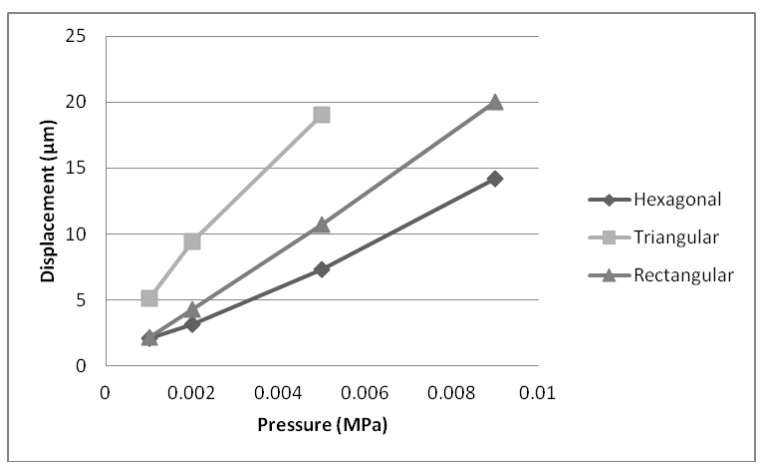

Fig 5: Pressure applied vs the displacement produced for the various shapes of SCR

\subsection{Thickness of cantilever}

The thickness was varied and was found the the displacement got saturated when the thickness was less than 5 microns and the sensitivity got reduced when thicker cantilever was used hence and optimum 10 micron thick cantilever was used.

\subsection{Positioning of Peizoresistor}

The stress concentration is found near the fixed end and hence the Peizoresistors were placed in various positions and the resistance formed was analysed and the optimum positioning is shown in Fig.6(a). Here for a $\mathrm{KPa}$ input change we could obtain a ohm of resistance change and hence a $\mathrm{mV}$ change in output, whereas the other placements gave only a microvolt change.

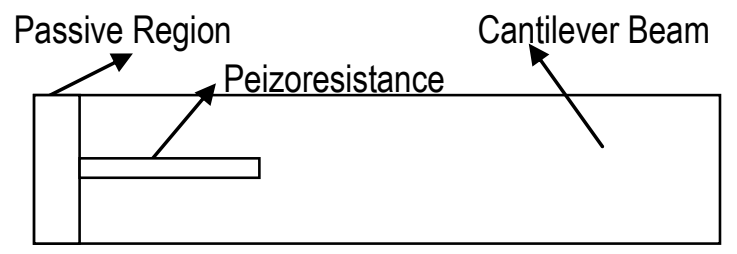

Fig 6(a): Peizoresistor placed perpendicular to the fixed plane

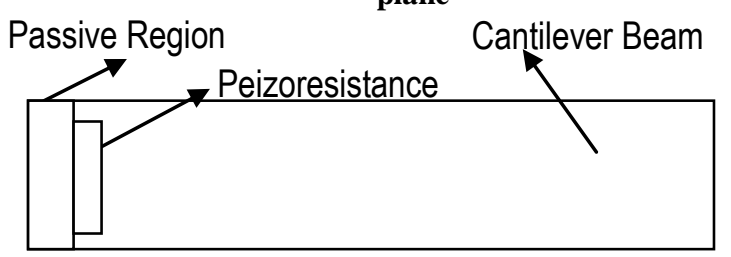

Fig 6(b): Peizoresistor placed along the fixed plane 


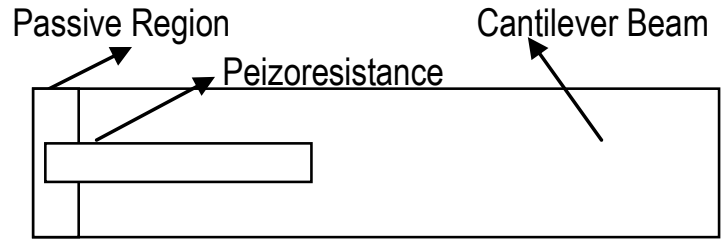

Fig 6(c): Peizoresistor embedded partially in the fixed plane

\subsection{Pressure - Displacement Curve}

The linear relation between the input and output is observed and is shown in Fig. 6

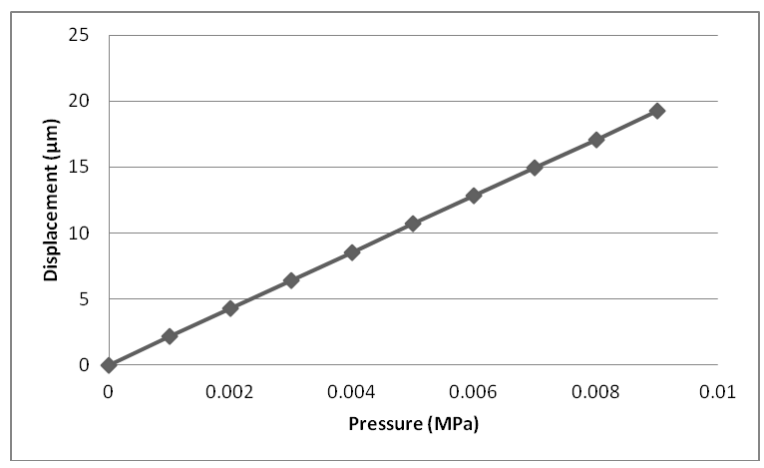

Fig 7: Input - Output relation of cantilever

\subsection{Pressure - Resistance Curve}

From Fig. 7 we can notice the linear relation between the input pressure and the peizoresistance change. Sensitivity is $840 \mathrm{ohm} / \mathrm{MPa}$.

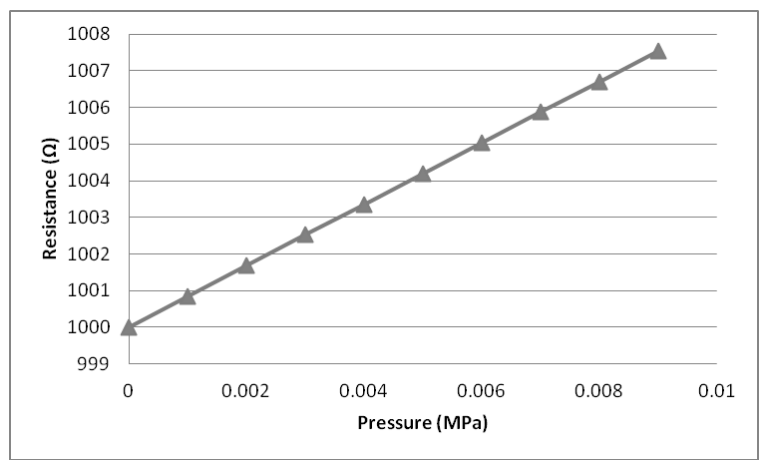

Fig 7: Input Pressure and the Peizoresistance Characteristics

\subsection{Pressure - Voltage Curve}

The ultimate output of the sensor is the output of the wheatstone bridge i.e the voltage. From Fig.8 it could be observed that there is a very good linear relation between the input pressure and the output voltage. The sensitivity is also in the range of $840 \mathrm{mV} / \mathrm{MPa}$. This is a good sensitivity range for a sensor.

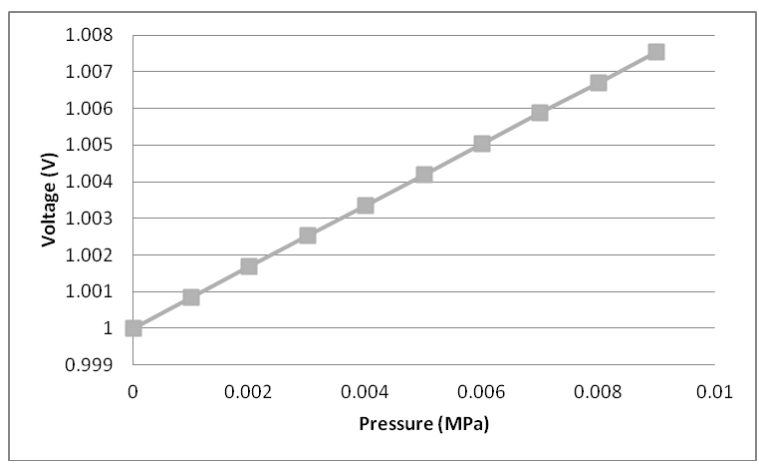

Fig 8: Input Pressure and the bridge output voltage Characteristics

\section{CONCLUSION}

We have thus designed a $\mathrm{CO} 2$ sensor having a cantilever with rectangular SCR for sensing and the thickness is 10micron and the mesh size used for analysis is 80 microns. A peizoresistor was incorporated in the most stressful area and the resistance change was studied using a bridge circuit and an equivalent voltage was obtained. The siumulated results were compared and studied. This sensor could prove to be a very effective handheld device if the noise patterns could also be removed by using suitable electronic circuits.

\section{REFERENCES}

[1] Surya, S.; Nag, S.; Fernandes, A.J.; Gandhi, S.; Agarwal, D.; Chatterjee, G.; Rao, V.R., 2011, Highly Sensitive _R/R Measurement System For Nano-Electromechanical Cantilever Based Bio-Sensors, 2011 International Symposium On Electronic System Design

[2] S.M. Firdaus I.A. Azid O. Sidek K. Ibrahim M. Hussien, 2010, Enhancing the sensitivity of a mass-based piezoresistive micro-electro-mechanical systems cantilever sensor, Published in Micro \& Nano Letters, Vol. 5, Iss. 2, pp. 85-90

[3] H.A. Mol, P.M. Sarro, H.Schellevis, Y.Hou, 2011, Process for low temperature deposition of Strain Gauge materials based on Chromium Nitride Thin Films, IEEE

[4] Raiteri R., Massimo G., Hans-Jurgen B., Petr S. 2001, Micomecahnical Cantilever-Based Biosensors, Sensor Actuator B, 79, Pp. 115-126

[5] Kouravand S., Ghader R., Mehdi S., Ahmadali T. 2006, Mems Capacitive Micro Thermometer Based On Tip Deflection Of Bimetallic Cantilever Beam, J. Sensor Transducers, 70, Pp. 637-644

[6 Brugger J., Despont M., Rossel C., Rothuizen H., Vettiger P., Willemin M., 1999, Microfabricated Ultrasensitive Piezoresistive Cantilevers For Torque Magnetometry, Sensors Actuators, 73, Pp. 235-242

[7] Jiang Y.G., Ono T., Eshashi M., 2008, Fabrication Of Piezoresistive Nanocantilevers For Ultra-Sensitive Force Detection, Meas. Sci. Technol., 19, Article No. 084011 (5 Pp)

[8] Calleja M., Nordstrom M., Alvarez M., Tamayo J., Lechuga L.M., Boisen A., 2005, Highly Sensitive Polymer-Based Cantilever-Sensors For Dna Detection, J. Ultramicrosc., 105, Pp. 215-222 
[9] Wang Z., Yue R., Zhang R., Liu L., 2005, Design And Optimization Of Laminated Piezoresistive Microcantilever Sensors, Sensors Actuators A, 120, Pp. 325-336

[10] Yu X., Zhang H., Li X., Li T., Zhang D., 2005, Design And Characterization Of High-Sensitivity Cantilevers, Proc. Ieee Conf. On Sensors, Hyatt Regency Irvine, Orange County, Irvine, Ca, Usa, Pp. 588-591

[11] He J.H., Li Y.F., 2006, High Sensitivity Piezoresistive Cantilever Sensor For Biomolecular Detection, J. Phys. Conf. Series, 34, Pp. 429-435

[12] Bhatti M.A., Lee C.X., Lee Y.Z., Ahmed N.A., 2007, Design And Finite Element Analysis Of Piezoresistive Cantilever With Stress Concentration Holes, Second Ieee Conf. On Industrial Electronics And Applications, Shangari-La Hotel, Harbin, People's Republic Of China, Pp. 1171-1174

[13] Arik M., Zurn S.M., Yigit K.S., Bar-Cohen A., 1999, Design, Fabrication And Experimental-Numerical Study Of Pzt Sensors, University Of Minnesota, Mn 55455.

[14] Chang L. , Foundation Of Mems, Illinois Ece Series, Pearson Education, Upper Saddle River, Nj, 2006

[15] Chu Duc T., Creemer J.F., Sarro Pasqualina M., 2006, Lateral Nano- Newton Force-Sensing Piezoresistive
Cantilever For Microparticle Handling', J. Micromech. Microeng., 16, Pp. 102-106

[16] Chu Duc T., Creemer J.F., Sarro Pasqualina M., 2007, Piezoresistive Cantilever Beam For Force Sensing In Two Dimensions, Ieee Sensors J., 7, (1), Pp. 96-104

[17] Fuller L.F., 2007, Microelectronic Engineering', Department Of Rochester, Institute Of Technology, Available At: Http:Www.Rit. Edu/ Iffee/,

[18] Jennifer C. Xu, Gary W. Hunter, Dorothy Lukco, ChungChiun Liu, And Benjamin J. Ward, 2009, Novel Carbon Dioxide Microsensor Based On Tin Oxide Nanomaterial Doped With Copper Oxide, Ieee Sensors Journal, Vol. 9 , No. 3.

[19] O. Pummakarnchana, V. Phonekeo, A. Vaseashta., 2007 A Real-Time Air Pollution Monitoring in Urban Environment Using In-Situ Measurements Using WO3 Gas Sensors and Satellite Imagery Through Internet GIS, Sensors \& Transducers Journal, Vol.77, Issue 3, pp.1065-1072 